\title{
The Use of Adverse Outcome Pathways (AOPs) to Support Chemical Safety Decisions Within the Context of Integrated Approaches to Testing and Assessment (IATA)
}

\author{
Catherine Willett $\left.{ }^{(}\right)$ \\ Animal Research Issues, Humane Society of the United States/Humane Society \\ International, 1255 23rd Street NW, Suite 450, Washington, D.C. 20037, USA \\ kwillett@humanesociety.org
}

\begin{abstract}
New streamlined approaches that use fewer resources and animals are needed for the safety assessment of chemicals. Data gathering should be streamlined to fit regulatory need and the specific properties of the chemicals being assessed. Toward this goal, the Organization for Economic Cooperation and Development (OECD) has introduced the concept of integrated approaches to testing and assessment (IATA) to inform hazard or risk assessment. An IATA is designed to address a specific question, and may include exposure or regulatory considerations, depending on the context. IATA can be informed by mechanistic information about the chemical and related biology. OECD's of Adverse Outcome Pathways (AOPs) framework is designed to gather biological information related to adverse outcomes of regulatory significance. An AOP is the collected chemical and biological information about a particular biological pathway. The OECD has developed guidance for building and assessing AOPs, and is coordinating development of the AOP Knowledge-base (AOP-KB) for collecting and using this information. The AOP-KB also accepts information about the perturbations of these pathways caused by chemical exposure information can be used to design prediction models. AOPs can form the logical basis for the integration of information and the design of integrated testing strategies (ITS), within the context of an (IATA), to more effectively and efficiently inform hazard or risk determination.
\end{abstract}

Keywords: Adverse Outcome Pathway (AOP) - Integrated Approach to Testing and Assessment (IATA) · Chemical safety assessment

New Approach Methodologies (NAM)

\section{Introduction}

There are scientific, social, economic, practical and regulatory pressures stimulating the development and use of streamlined chemical testing and assessment approaches, including increased reliance on non-animal approaches [also called new approach methodologies (NAM)]. Advances in biological understanding as well as in experimental technologies (e.g. 'omics tools, cell culturing, reconstructed tissues) have allowed the consideration of dramatically different approaches to chemical safety 
assessment than those traditionally practiced. Increasingly, combinations of non-testing and non-animal test methods are replacing apical animal tests.

For example, there are perhaps tens of thousands of industrial chemicals already in use and in the environment about which there is little toxicological information, and many new chemicals are developed each year, necessitating implementation of a new approach that can broadly assess a large number of chemicals relatively quickly. Another driver comes from the personal care product sector where consumers and regional laws are increasing pressure to develop products without testing on animals. To build a more efficient process, a 2007 National Research Council (NRC) report recommends "Transform(ing) toxicity testing from a system based on whole animal testing to one founded primarily on in vitro methods that evaluate changes in biologic processes using cell, cell lines, or cellular components, preferably of human origin" (NRC 2007).

\section{The Adverse Outcome Pathway (AOP) Framework}

In order to be able to make decisions using this new type of information, we need to understand how changes at the molecular level in cells and tissues are related to the apical adverse outcomes (such as tumor development, changes in organ size or histology, or death) that toxicologists are more familiar with Fig. 1). The Organization of Economic Cooperation and Development (OECD) has created the Adverse Outcome Pathway framework to collect, organize and evaluate the biological information that links upstream molecular changes with downstream organ, organism and population changes (Fig. 1). According the OECD AOP handbook, AOPs "can be viewed as a sequence of events commencing with initial interactions of a stressor with a biomolecule in a target cell or tissue (i.e., molecular initiating event), progressing through a dependent series of intermediate events and culminating with an adverse outcome... AOPs are typically represented sequentially, moving from one key event to another, as compensatory mechanisms and feedback loops are overcome" (OECD 2018).

Every AOP consists of a variable number and arrangement of a few key elements: a molecular initiating event (MIE), the adverse outcome (AO) of regulatory relevance, any number of intermediate Key Events (KE) and the relationships between these elements (key event relationships or KERs). Five general principles are involved in AOP development: (1) AOPs are not chemical specific, (2) AOPs are modular (consisting of KEs and KERs) that can be shared between two or more pathways, (3) An individual AOP is a pragmatic unit of development and evaluation; (4) for most realworld applications, AOP networks are the functional unit of prediction, and (5) AOPs are living documents, and, as more information is discovered, will be continuously updated and never really finished (Villeneuve et al. 2014). OECD is coordinating the development of the software (the AOP knowledge-base or AOP-KB) as well as a number of guidance documents to facilitate the development, evaluation and use of AOPs. In addition, the Human Toxicology Project Consortium (which is coordinated by the Humane Society of the United States), in collaboration with OECD's AOP program, has created a training course that is freely available to anyone. These 


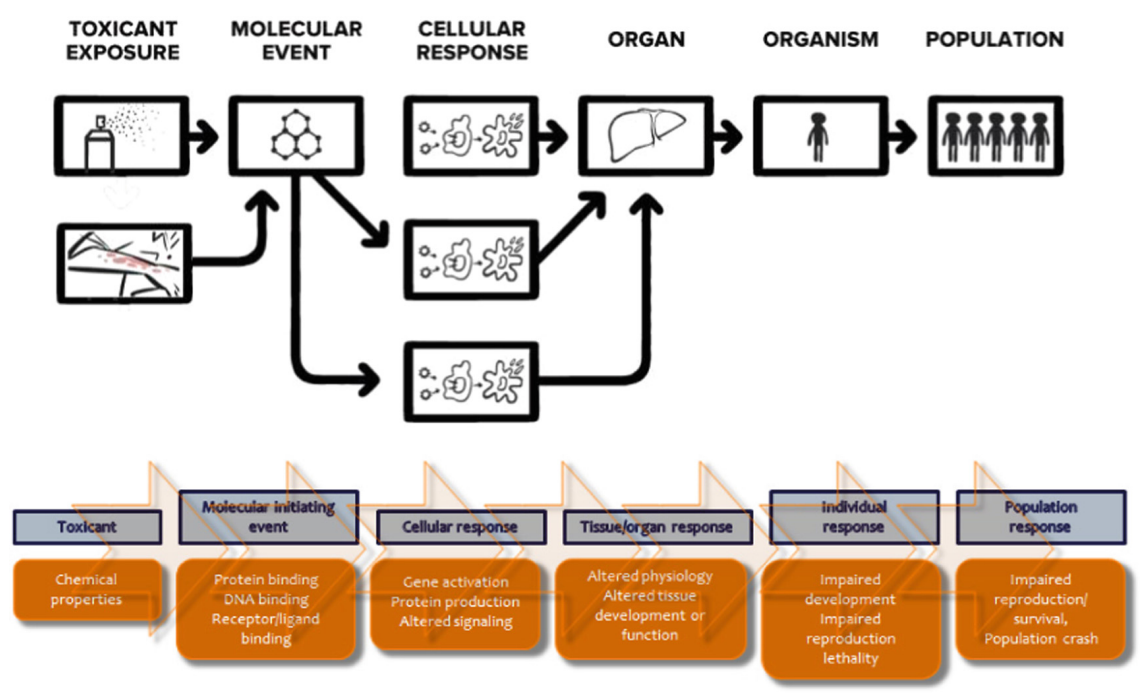

Fig. 1. Linking upstream molecular changes to adverse outcomes: the Adverse Outcome Pathway (AOP) project of the Organization for Economic Cooperation and Development (OECD). (Diagram from "Pathways to a Better Future" video series, (C) The Human Toxicology Project Consortium).

documents are available from the OECD website, and the training course is available from the HTPC website or at https://aopwiki.org. ${ }^{1}$

The main element of the AOP-KB is currently the AOP Wiki (https://aopwiki.org). The AOP wiki is designed to capture all of the information about AOP elements in text form, via standardized drop-down menus (to keep a standard format and consistent ontology) and free text fields (to accommodate data, explanations and references). As of this writing, there are more than 200 AOPs and more than 1000 KEs and KERs described in the wiki linked to 340 different stressors (metrics available on the AOP Wiki). As more pathways are described, connections between KEs that are common to different pathways are identified, and in this way, biological networks, which are the basis for predicting AOs from MIEs, are discovered.

The AOP Wiki also supports evaluation of the consistency, quantity and quality of the information supporting the KERs and AOP overall (Table 1). The criteria for evaluating KERs and AOPs, modified from the Bradford-Hill criteria for evaluating causal linkages are described in the OECD guidance and in Meek et al. 2014. AOPs that are published in the OECD program series are evaluated in two stages. The first review is by members of the OECD AOP program to make sure the information has been entered in compliance with OECD guidance. The second stage is an independent scientific review by a subject matter expert group that can be a combination of OECD

${ }^{1}$ OECD AOP Program: http://www.oecd.org/chemicalsafety/testing/adverse-outcome-pathways-mole cular-screening-and-toxicogenomics.htm; HTPC training course: https://humantoxicologyproject. org/about-pathways-2/aop-online-course/. 
Table 1. Biological plausibility: between KE upstream and KE downstream?

\begin{tabular}{|c|c|c|}
\hline $\begin{array}{l}\text { High (strong): Extensive } \\
\text { understanding of KER }\end{array}$ & Moderate: KER is plausible & $\begin{array}{l}\text { Low (weak): some empirical } \\
\text { support }\end{array}$ \\
\hline \multicolumn{3}{|c|}{ Essentiality: are downstream KEs prevented if upstream KE's blocked? } \\
\hline $\begin{array}{l}\text { High (strong): direct evidence } \\
\text { from experimental studies }\end{array}$ & Moderate: indirect evidence & $\begin{array}{l}\text { Low (weak) } \\
\text { No or contradictory evidence }\end{array}$ \\
\hline \multicolumn{3}{|c|}{ Empirical Evidence: amount, quality, consistent, inconsistent? } \\
\hline $\begin{array}{l}\text { High (strong): extensive } \\
\text { evidence for temporal, dose- } \\
\text { response }\end{array}$ & $\begin{array}{l}\text { Moderate: multiple reports of } \\
\text { consistent evidence, some } \\
\text { inconsistent }\end{array}$ & $\begin{array}{l}\text { Low (weak): limited or no studies } \\
\text { and/or significant inconsistencies }\end{array}$ \\
\hline
\end{tabular}

Extracted from OECD (2018), Annex I.

and outside experts - this is similar to peer review of a scientific publication. Once the authors and reviewers are satisfied, a "snapshot" of the AOP at that time is published on the OECD website and is considered the current state of knowledge about that pathway.

\section{The Use of AOPs to Support Hazard and Risk Assessment}

The AOP framework has been developed to facilitate the use of molecular and cellbased information to inform regulatory decisions. AOPs on their own are nothing more than organized and, in some cases, evaluated information; however, they can be used in several different ways to support chemical assessment. For example, AOPs can be used to support weight-of-evidence (WoE) evaluation of new or existing information (as is current performed during hazard and risk evaluations), they can be used to generate hypothesis to develop new information for answering a specific regulatory question (as in the context of an integrated approach to testing and assessment, IATA, described in more detail below), or if enough quantitative information is available, they can be used to create predictive models.

To streamline AOP-supported chemical assessment, OECD has also provided guidance for developing and assessing IATA. ${ }^{2}$ OECD guidance on developing IATA defines IATA as "pragmatic, science-based approaches for chemical hazard or risk characterization that rely on an integrated analysis of existing information in a weight of evidence assessment coupled with the generation of new information using testing strategies...IATA follow an iterative approach to answer a defined question in a specific regulatory context..." (Sachana and Leinala 2017; OECD 2016b). The IATA framework is flexible and allows streamlining of the testing and assessment process to generate exactly the information needed to make a decision. IATA can be as simple or as complex as needed to answer the specific question, depending on the amount of certainty needed.

AOPs can support IATA development and implementation in a number of ways (Fig. 2). AOPs can support WoE evaluation of existing data and, in cases where more

\footnotetext{
${ }^{2}$ OECD Integrated Approaches to Testing and Assessment: http://www.oecd.org/chemicalsafety/riskassessment/iata-integrated-approaches-to-testing-and-assessment.htm.
} 


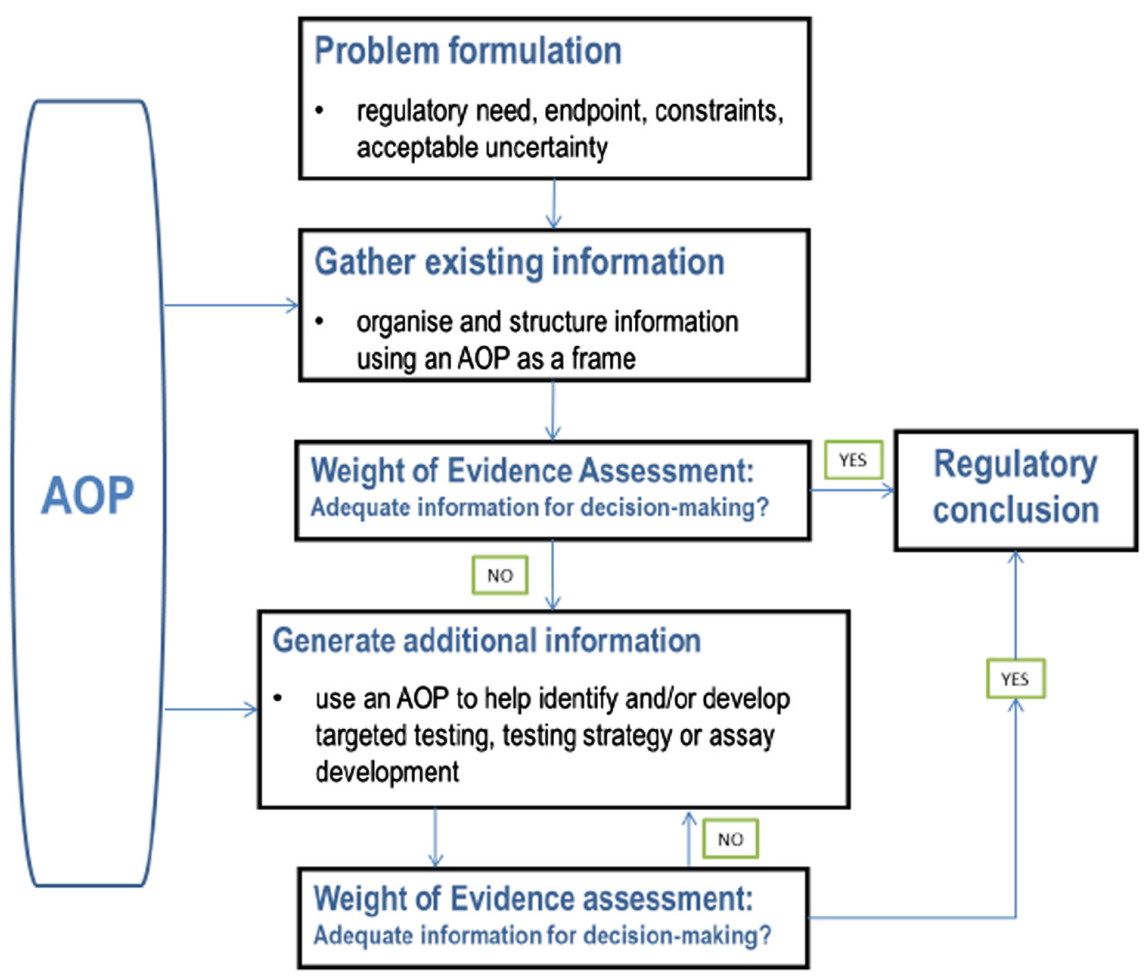

Fig. 2. AOPs can support Integrated Approaches to Testing and Assessment (IATA). (Diagram reprinted from OECD (2016a).

information is needed, identify what information would decrease uncertainty and suggest the method by which to obtain that information. AOPs can be consulted iteratively until sufficient information is obtained to answer the question with the required certainty.

While IATA are designed to be flexible, tailored approaches to address regulatory needs, the potential variability in IATA can cause uncertainty for regulators as well as the regulated community. If there are several different ways to answer the same regulatory question, how do regulators know that each are equally reliable, and how can the submitters of the data be assured of regulatory acceptance? To address this potential regulatory issue, individual standardized IATA, with defined methods and interpretation procedures can be evaluated for regulatory application. OECD has published guidance to facilitate standardization and evaluation of IATA (OECD 2016b). According to this guidance, there are six general principles that are included in every IATA: the defined approach should be associated with the following set of information: (1) a defined endpoint, (2) a defined purpose, (3) a description of the underlying rationale, (4) a description of the individual information sources used, (5) a description of how data from the individual information sources are processed, (6) a consideration of the known uncertainties (and in each case, a description of the magnitude of each source of uncertainty). 


\section{AOP-Supported IATA Case Study: Skin Sensitization}

The AOP for skin sensitization has been well-described, and several assays have been identified that can query the MIE and upstream KEs, and there is quite a bit of evidence substantiating the prediction of the AO by measuring various combinations of these KEs (OECD 2012a, b). There are also a number of different IATA that have been developed to assess skin sensitization (e.g. Natsch et al. 2013; Ramirez et al. 2016; OECD 2016c) (Fig. 3). Nearly all sensitizers are molecules that are or can become electrophiles and will react covalently with proteins. If these substances are exposed to and can penetrate skin, they are likely to cause allergic reactions in humans. Several computer models exist that relate the structure of a molecule to its potential to become an electrophile (Quantitative Structure Activity Relationships or QSARs), and for many molecules, can accurately predict skin sensitizing potential. Several QSARs and in vitro test methods exist to look at the MIE (covalent reaction with proteins) and two early KE (dendritic cell and keratinocyte activation). IATA using combinations of one or more of these assays have been evaluated for regulatory application, some of which outperform animal tests to predict human skin sensitization (Bauch et al 2012; Natsch et al. 2013; OECD 2016c).

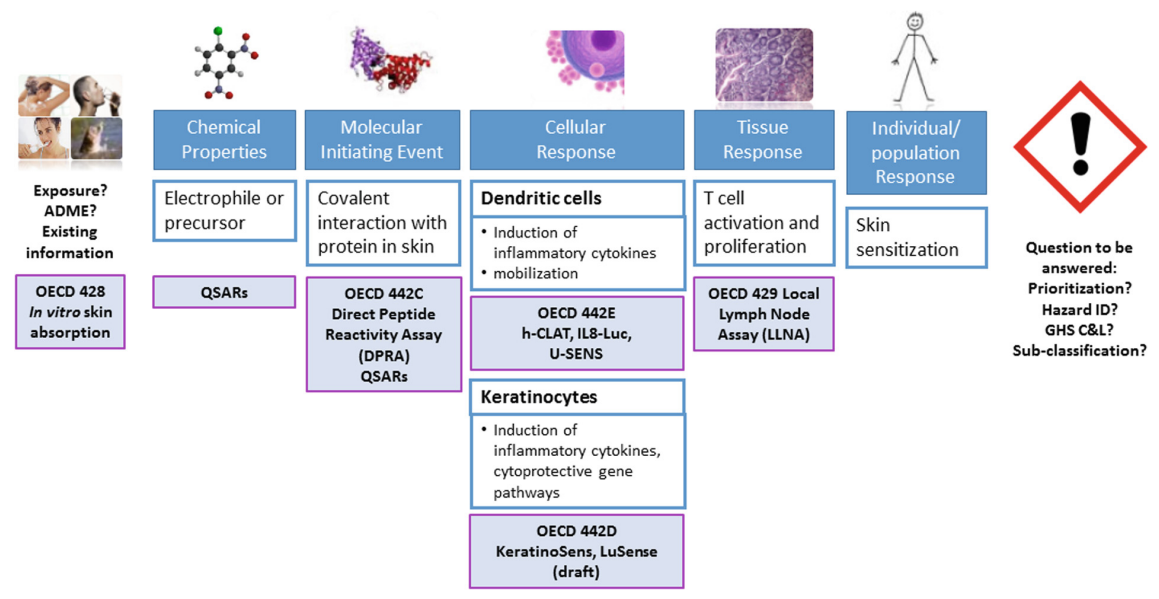

Fig. 3. Skin sensitization AOP and validated methods to assess various KEs (pink outlined boxes).

To facilitate regulatory uptake of IATA for skin sensitization, the International Cooperation on Alternative Test Methods (ICATM), in collaboration with OECD and the European Commission's Joint Research Centre, held a workshop on the International Regulatory Applicability and Acceptance of Alternative Approaches to Skin Sensitization Assessment of Chemicals (Kleinstreuer et al. 2017). Attendees, which included 40 regulators and scientists from 12 countries, concluded that in vitro methods are available that are adequate for use within an IATA to predict skin sensitization for regulatory purposes, but that a number of barriers remain to be surmounted, including: a lack of 
familiarity and need for training in the methods and development of clear guidance for application of multiple IATA. A recommendation was for OECD to develop a performance-based test guideline for skin sensitization IATA. To follow on this workshop, the OECD is coordinating a project, led by the US ICCVAM and Environmental Protection Agency and the EC JRC, to evaluate the 12 IATA described in OECD Guidance Document 256 (OECD 2016c). A special OECD workshop was held in Dec 2017 to review the progress and obtain input from the OECD national coordinators of the test guideline program. Results of the study are expected in mid 2018.

\section{Summary}

AOPs can support decision making, including for regulatory application, and in several ways. AOPs can: support WoE evaluations; form the basis for integrated approaches to testing and assessment; provide the basis for biological hypothesis to identify what information would be most helpful for decreasing uncertainty in a given situation; provide transparent communication of the information and uncertainty that underlie decisions; form the basis for development of predictive models. The OECD is coordinating an $\mathrm{AOP} \mathrm{KB}$ for collecting and assessing information related to AOPs. The AOP Wiki is a crowd-sourcing platform that is open to all interested parties and guidance materials and training courses to assist in use are freely available. Participation in the wiki should be promoted as the more expert input into the wiki, the more useful the knowledgebase will become. IATA are designed to streamline regulatory decision making. OECD has created a number of guidance documents to assist with the development and review of IATA. Development of IATA begin with problem formulation that includes consideration of the regulatory context and gathering of all existing relevant information. IATA are hypothesis-driven and can be supported by AOPs. There can be more than one IATA to answer a given question, and that necessitates the development of specific cases of IATA, or defined approaches, that consist of defined methods and data interpretation procedures, for regulatory application. OECD and member organizations are developing case studies of defined approaches, beginning with skin sensitization.

\section{References}

Bauch C, Kolle SN, Ramirez T, Eltze T, Fabian E, Mehling A, Teubner W, van Ravenzwaay B, Landsiedel R (2012) Putting the parts together: combining in vitro methods to test for skin sensitizing potentials. Regul Toxicol Pharmacol 63(3):489-504. https://doi.org/10.1016/j. yrtph.2012.05.013. (Erratum in: Regul Toxicol Pharmacol 64(2):285 2012)

Kleinstreuer N, Strickland J, Casati S, Barroso J, Zuang V, Lowit A, Matheson J, Allen D, Casey W, Whelan M (2017) Evaluating defined approaches to testing and assessment of skin sensitization potential. Presented at the society of toxicology annual meeting, March 12-16, Baltimore, MD. https://ntp.niehs.nih.gov/iccvam/meetings/sot17/kleinstreuer-sotposter1-fd. pdf. Accessed 31 Jan 2018

Meek ME, Palermo CM, Bachman AN, North CM, Lewis RJ (2014) Mode of action human relevance $(\mathrm{MOA} / \mathrm{HR})$ framework-evolution of the bradford hill considerations and comparative analysis of weight of evidence. Toxicol, Appl. https://doi.org/10.1002/jat.2984 
National Research Council (2007) Toxicity testing in the 21st century: a vision and a strategy. National Academy of Sciences, Washington, DC, USA

Natsch A, Ryan CA, Foertsch L, Emter R, Jaworska J, Gerberick F, Kern P (2013) A dataset on 145 chemicals tested in alternative assays for skin sensitization undergoing prevalidation. J Appl Toxicol 33:1337-1352. https://doi.org/10.1002/jat.2868

OECD (2012a) The adverse outcome pathway for skin sensitisation initiated by covalent binding to proteins, part 1: scientific evidence. Series on Testing and Assessment, No. 168. OECD, Paris, France

OECD (2012b) The adverse outcome pathway for skin sensitisation initiated by covalent binding to proteins, part 2: use of the AOP to develop chemical categories and integrated assessment and testing. Series on Testing and Assessment, No. 168. OECD, Paris, France

OECD (2018) Users' Handbook supplement to the guidance document for developing and assessing adverse outcome pathways. OECD Series on Adverse Outcome Pathways, No. 1, OECD Publishing, Paris. http://dx.doi.org/10.1787/5jlv1m9d1g32-en

OECD (2016a) Reporting of defined approaches to be used within integrated approaches to testing and assessment. Series on Testing and Assessment, No. 255. OECD Publishing, Paris

OECD (2016b) Use of adverse outcome pathways in developing integrated approaches to testing and assessment (IATA). Series on Testing and Assessment, No. 260. OECD Publishing, Paris

OECD (2016c) Guidance document on the reporting of defined approaches and individual information sources to be used within integrated approaches to testing and assessment (IATA) for skin sensitisation. Series on Testing and Assessment, No. 256. OECD Publishing, Paris

Ramirez T, Stein N, Aumann A, Remus T, Edwards A, Norman KG, Ryan C, Bader JE, Fehr M, Burleson F, Foertsch L, Wang X, Gerberick F, Beilstein P, Hoffmann S, Mehling A, van Ravenzwaay B, Landsiedel R (2016) Intra- and inter-laboratory reproducibility and accuracy of the LuSens assay: a reporter gene-cell line to detect keratinocyte activation by skin sensitizers. Toxicol Vitr 32:278-286

Sachana M, Leinala E (2017) Approaching chemical safety assessment through application of integrated approaches to testing and assessment: combining mechanistic information derived from adverse outcome pathways and alternative methods. Appl Vitr Toxicol 3(3): 227-233

Villeneuve DL, Crump D, Garcia-Reyero N, Hecker M, Hutchinson TH, LaLone CA, Landesmann B, Lettieri T, Munn S, Nepelska M, Ottinger MA, Vergauwen L, M Whelan (2014) Adverse outcome pathway (AOP) development I: strategies and principles. Toxicol Sci 142:312-320

Open Access This chapter is licensed under the terms of the Creative Commons Attribution 4.0 International License (http://creativecommons.org/licenses/by/4.0/), which permits use, sharing, adaptation, distribution and reproduction in any medium or format, as long as you give appropriate credit to the original author(s) and the source, provide a link to the Creative Commons licence and indicate if changes were made.

The images or other third party material in this chapter are included in the chapter's Creative Commons licence, unless indicated otherwise in a credit line to the material. If material is not included in the chapter's Creative Commons licence and your intended use is not permitted by statutory regulation or exceeds the permitted use, you will need to obtain permission directly from the copyright holder.

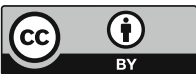

